\title{
Modeling of the Feed-Motor Transient Current in End Milling by Using Varying-Coefficient Model
}

\author{
Mi Xiao, ${ }^{1}$ Long Wen, ${ }^{1} \mathrm{Xi} \mathrm{Li}^{2}$ and Liang Gao ${ }^{1}$ \\ ${ }^{1}$ State Key Laboratory of Digital Manufacturing Equipment and Technology, Huazhong University of Science and Technology, \\ Wuhan 430074, China \\ ${ }^{2}$ School of Mechanical Science and Engineering, Huazhong University of Science and Technology, Wuhan 430074, China
}

Correspondence should be addressed to Long Wen; longwen12@gmail.com

Received 4 July 2014; Accepted 16 October 2014

Academic Editor: Ivanka Stamova

Copyright (C) 2015 Mi Xiao et al. This is an open access article distributed under the Creative Commons Attribution License, which permits unrestricted use, distribution, and reproduction in any medium, provided the original work is properly cited.

\begin{abstract}
In order to ensure the stability of the machining process, it is vital to control the machining condition during the milling process. While the feed-motor current is related to many physical variables, such as the cutting force and tool wear, we can indicate it as the key variables to monitoring the conditions of the milling process. A predictive model of the feed-motor current amplitude is established in this paper. The change regulation of the transient current amplitude during the milling process is investigated, and the effect of the spindle speed on the transient current amplitude is studied as well. Since the transient current amplitude is time-varying, the predictive model is a typical panel data type. In this case, the varying-coefficient model (VCM), a potential soft computing method, is applied to solve this predictive model. Then several experiments are conducted to evaluate the performance of VCM method. Results show that the predicted values match the experimental value well, and the correctness of the predictive model for transient current amplitude is also validated.
\end{abstract}

\section{Introduction}

High-speed machining (HSM) has shown a lot of advantages in the manufacturing industries with the development of the computer numerical control (CNC) systems. It provides a way to achieve a higher quality of the work-piece and can increase the productivity of the machining process. However, due to the complexity of the machining process, the control of HSM to ensure the stability during the machining process is a challenging work.

Many researchers focus on the modelling of the machining process and the physical variables, such as cutting force [1], chatter [2], and tool wear [3], in order to monitor the machining process. In recent years, soft computing methods have been applied to the modeling and prediction in the machining field [4], and their potential is validated. Surface roughness, cutting force, tool life, and the material remove rate are the most common machining physical variables. Moghri et al. [5] applied the artificial neural network (ANN) to predict the surface roughness in milling of ployamide- 6 nanocomposites. Ren et al. [6] presented a subtractive clustering-based fuzzy approach method for modelling the cutting force in micromilling; the obtained results proved that the proposed solution can model the cutting force in spite of uncertainties in the micromilling process. Gokulachandran and Mohandas [7] compared neurofuzzy logic technique and support vector regression technique for the assessment of remaining useful life of cutting tools and obtained good results.

As the machining parameters are easier to control compared with other input, the effect of the machining parameters to the physical variables is critical to monitor the machining process, and the application of soft computing method to model the relationship between the machining parameters and machining process is increasing. But most machining physical variables are nonlinear and random, making it difficult to model the machining process. Cakir et al. [8] tried to establish the quantitative relationship between the process parameters and the performance of electrical discharge machining (EDM) process using adaptive neurofuzzy inference system (ANFIS), genetic expression programming 
(GEP), and ANN. The result shows that all approaches are successful in the prediction of EDM performance. Zohourkari et al. [9] investigated the effects of the machining parameters on the material removal rate in the abrasive waterjet turning process using analysis of variance (ANOVA), and the result is good. Sharkawy [10] presented the study of modeling the surface roughness in end milling using radial basis function neural networks (RBFNs), ANFIS, and genetically evolved fuzzy inference systems (G-FISs), and the prediction accuracy is as high as $97.05 \%$.

Modeling and controlling the machining process using soft computing have achieved a great success. However, even though several machining physical properties have been studied, the research on the field of the feed-motor current remains limited. The feed-motor current is one of the most vital machining variables in the milling process. Many other machining physical variables, such as cutting force and tool wear, are related to the feed-motor current. Aggarwal et al. [11] identified the cutting torque and tangential cutting force coefficient from the spindle motor current, Jeong and Cho [12] estimated the cutting force from the rotating and stationary feed-motor current, and Rizal et al. [3] detected the tool breakage in CNC high-speed milling based on the feed-motor current. Kim and Jeon [13] show that feedmotor is better for the control of the CNC milling process than cutting forces. Thus, the research on the feed-motor current is very essential in order to understand and control the machining process. Xu and Jie [14] studied the current empirical equation between the effective values of feed-motor current and the machining parameters. Even though this empirical equation fits the experiments well, the law of the feed-motor current lacks.

In this research, the feed-motor current is studied using soft computing method. A quantitative prediction model of the feed-motor transient current amplitude is established in the end milling process. The transient current is time-varying during the milling process, making it different from the traditional current empirical equation. The transient current predictive model is extended from this empirical equation. The change regulation of the feed-motor transient current is investigated in the predictive model, as well as the effect of the spindle speed to the feed-motor current. As the feedmotor transient current is time-varying, the transient current predictive model is a typical panel data in this study. Varyingcoefficient model (VCM) is applied to solve the transient current predictive model. Several experiments are conducted to evaluate the correctness of the predictive model and the performance of the VCM methods; the result shows that the predictive results match the experimental value very well.

The rest of this paper is organized as follows. In Section 2, the predictive model of the transient current is established. The varying-coefficient model is shown in Section 3. The experiments and results are presented in Section 4. The conclusion is drawn in Section 5.

\section{The Predictive Model of the Transient Current}

The experiments are carried out on a machining center (OTM-650), the work-piece material is the 45 steel and the tool material is high-speed steel, and the number of the tool teeth is three. During the experiments, the spindle speed levels are 500, 600, 700, 800, 1000, and $1200 \mathrm{rpm}$. The feed speed is $200 \mathrm{~mm} / \mathrm{min}$ and the axial depth of cut is $0.2 \mathrm{~mm}$ in all experiments. There are 10 repeated experiments under the different spindle speed level. As shown in Figure 1, two phases of the feed-motor current, namely, the $U$ phase $I_{u}$ and the $V$ phase $I_{v}$, are sampled during the milling process, and the sampling frequency is $512 \mathrm{~Hz}$.

2.1. Data Acquisition. During the milling process, the $U$ phase $I_{u}$ and the $V$ phase $I_{v}$ of the feed-motor transient current are acquired and they are enough to calculate the amplitude $I$ of the three-phase feed-motor current. The relationship between them is

$$
\begin{aligned}
I_{u} & =I \cos \left(\omega_{1} t\right), \\
I_{v} & =I \cos \left(\omega_{1} t+\frac{2 \pi}{3}\right), \\
I & =\sqrt{\frac{4}{3}\left(I_{u}^{2}+I_{v}^{2}+I_{u} I_{v}\right)},
\end{aligned}
$$

where $\omega_{1}$ stands for the motor frequency, which is related to the spindle speed $S$, and $\omega_{1}=S / 22.5$.

Figure 2 shows the samples of the feed-motor transient current amplitude and indicates that the current amplitude is periodically changed, and the cycle is stable. Some feature values can be extracted in order to reduce the data volume of the transient current without losing its properties.

\subsection{Feature Values of the Transient Current}

2.2.1. Feature Values. Two feature values are extracted to represent the transient current amplitude, and they are the cycle and the starting point of the transient current. These two feature values are shown as in Figure 3. The cycle is the most important feature of the transient current amplitude. However, the start point is also vital and essential in order to determine the starting phase position of the transient current amplitude. With the help of feature values, it provides a more convenient way for the comparison process, and the analytical results made in one cycle are suitable for other cycles too.

The calculation of the two feature values is based on the Fourier series. The Fourier series is shown as follows:

$$
F(x)=a_{0}+\sum_{i=1}^{n}\left(a_{i} \cos (\omega x)+b_{i} \sin (\omega x)\right) .
$$

The cycle $T=2 \pi / \omega$, and the start phase is set to $-\pi / 2$. Here, frequency $\omega$ is a feature of transient current amplitude, and it has no association with the motor frequency $\omega_{1}$. As shown in Figure 3, the 2nd Fourier series is preferred.

2.2.2. Normalization Process. Even though the starting point is used to simplify the comparison of the transient current amplitude, the normalization process is needed. The milling process is disturbed by many factors, and there is a subtle 
TABLE 1: The cycle of the transient current amplitude with spindle speed $500 \mathrm{rpm}$.

\begin{tabular}{lcccccccccc}
\hline Number & 1 & 2 & 3 & 4 & 5 & 6 & 7 & 8 & 9 & 10 \\
\hline$\omega$ & 23.25 & 23.25 & 23.25 & 23.24 & 23.26 & 23.25 & 23.25 & 23.26 & 23.26 & 23.24 \\
\hline
\end{tabular}

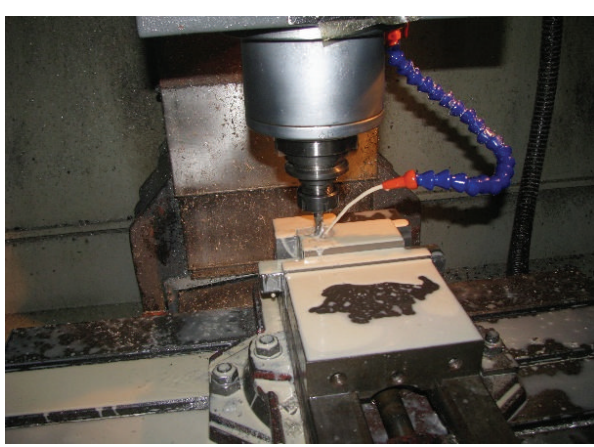

(a)

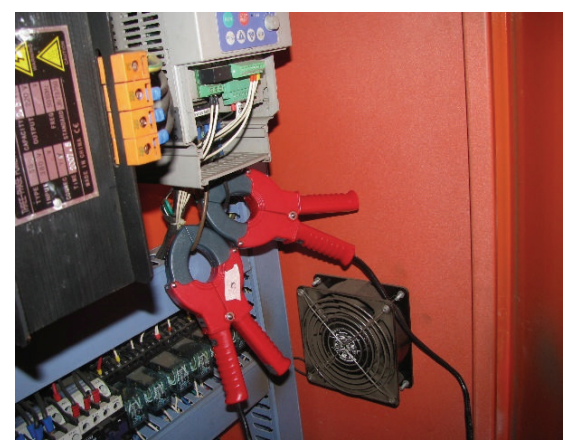

(b)

Figure 1: The experiment setup.

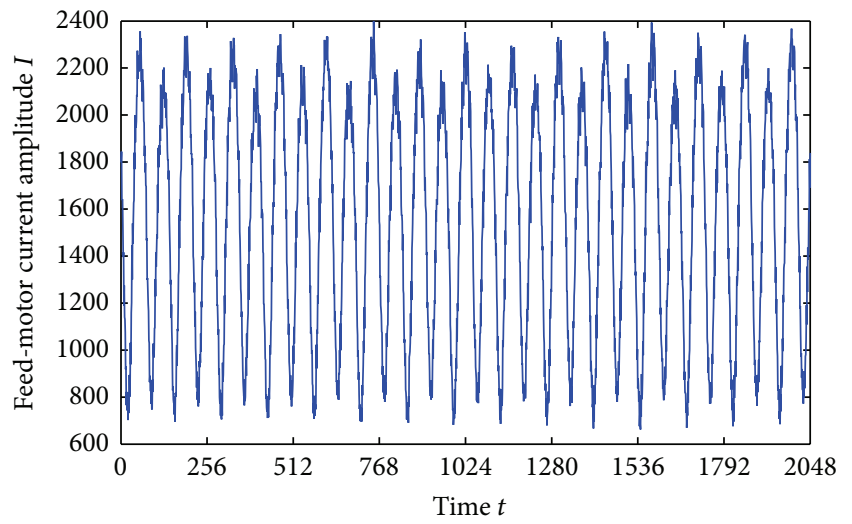

FIGURE 2: The feed-motor transient current amplitude samples with spindle speed $500 \mathrm{rpm}$.

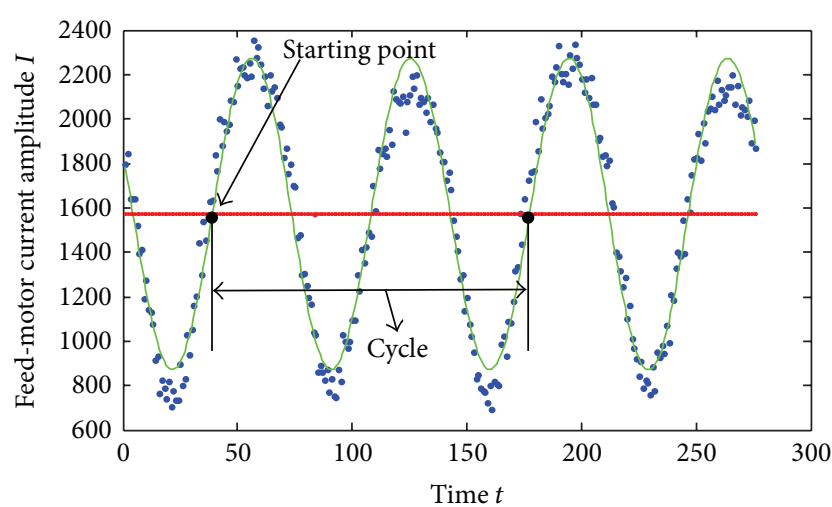

FIGURE 3: The feature values of the transient current amplitude.

difference between the results of feature values of 10 repeated experiments; even the machining parameters are the same for the repeated experiments.
Table 1 presents the frequency results of 10 repeated experiments using Fourier series. The result shows that they are slightly different from each other. In order to unify the 10 repeated experiments, all cycles are normalized to 1 to eliminate the disturbance.

2.2.3. The Validation Process. The validating process here is to check the effectiveness of the feature values and the normalization process. There are two testing parts in this section. The first one is used for testing the correctness of the feature values, and the second one is to test the validity of the normalization process.

The first testing part is the shift operator. In this operator, the samples in the later four cycles are shifted to the first cycle. The shifted result is shown in Figure 4(a); the black, green, red, pink, and blue samples came from different cycles. The samples in these five cycles are mixed very well, indicating the correctness of the feature values. It also can be inferred that the analytical result in one cycle is suitable for other cycles too.

The second testing part is conducted to test the normalization process. In this round, the cycle is set to 0.5 and the samples are selected on the first two cycles in all 10 repeated experiments with the spindle speed of $500 \mathrm{rpm}$. As shown in Figure 4(b), all samples are mixed together, and the mixed result is a smooth heavy line. The result indicates that the normalization process matches the experimental data well, meaning the analytical results of the mixed samples are suitable for all 10 repeated experiments.

2.3. Transient Current Predictive Model. The feature values show the change regulation of the transient current amplitude during the milling process. In this section, the relationship of the transient current amplitude and the spindle speed is considered, and the predictive model is established. 


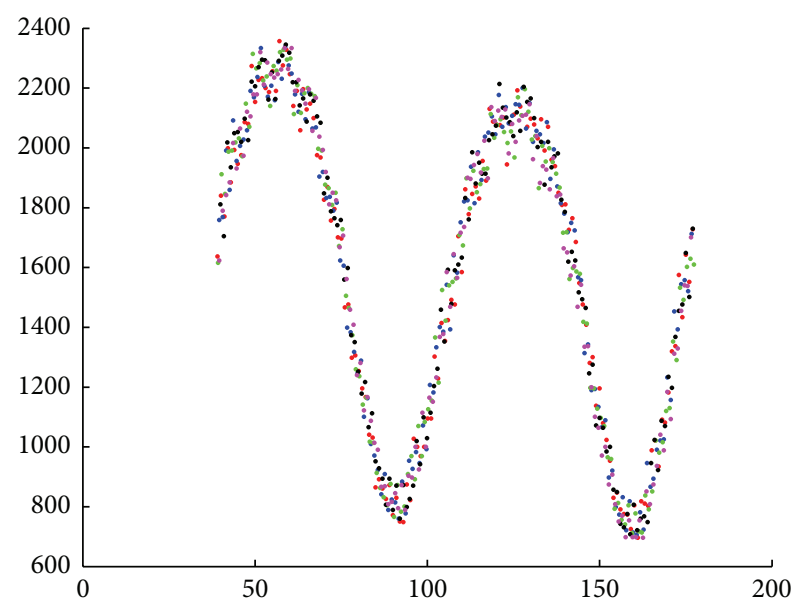

(a) The result of first testing part

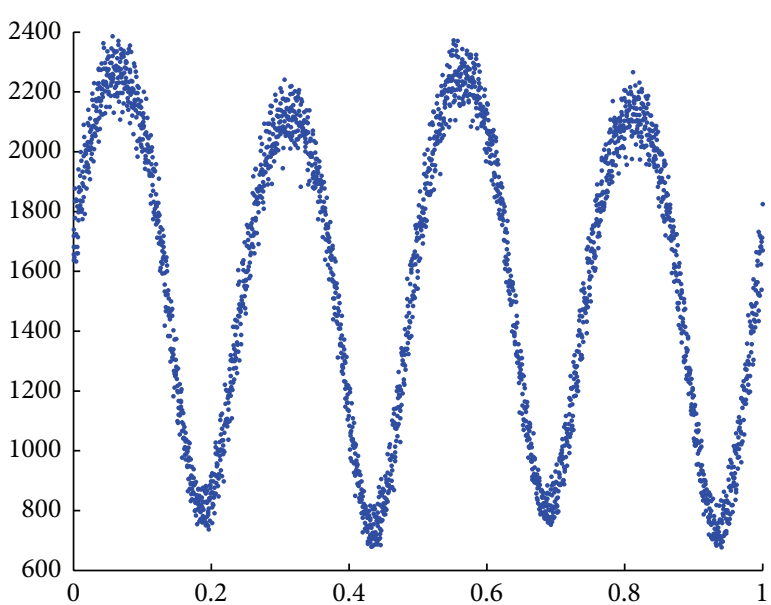

(b) The result of second testing part

FIGURE 4: The validation process of the transient current amplitude with spindle speed $500 \mathrm{rpm}$.

The traditional relationship between the feed-motor current and the machining parameters is an empirical equation, shown in (3). $I_{e}$ is the effective value of the feed-motor current, $S, f, D$ are the spindle speed, feed, and depth of cut, and $K_{0}, b_{1}, b_{2}, b_{3}$ are the coefficient parameters. Consider

$$
I_{e}=K_{0} S^{b_{1}} f^{b_{2}} D^{b_{3}}
$$

The transient current amplitude is different from the effective value of current; the former one is changing with time during the machining process. As presented in Section 2.2, the transient current amplitude is changing periodically during the milling process, and the cycle is stable. Moreover, the transient current amplitude is also related to the machining parameters, namely, the spindle speed. In order to consider the dynamic properties of the current amplitude, transient current predictive model extended the empirical equation as follows:

$$
I(t)=K_{0}(t) S^{b_{1}(t)}
$$

The transient current amplitude $I(t)$ and coefficient parameters $K_{0}$ and $b_{1}$ are extended to the function of time, in order to show that the transient current amplitude is timevarying during the stable milling process. The spindle speed affects the transient current amplitude as well in this model. For simplification, the predictive model can be transferred to a linear equation:

$$
\ln I(t)=\ln K_{0}(t)+b_{1}(t) \ln S .
$$

The simplified predictive model is a typical panel data type. The current amplitude is affected by the time and the spindle speed, which is the cross-sectional data type. Varyingcoefficient model (VCM) is very suitable to handle with panel data, so the VCM method is applied to solve this predictive model.

\section{Varying-Coefficient Model}

The traditional statistical model uses sample data $\left(X_{i}, Y_{i}\right)$ to estimate a regression function in which the response variable $Y$ is represented as a function of the predictor variable $X$, where $Y$ is one-dimensional and $X$ is a $p$-dimensional vector $\left(X=\left(X_{1}, X_{2} \cdots X_{p}\right)\right)$. Usually a linear relationship between $Y$ and $X$ is assumed:

$$
Y_{j}=\beta_{j} X_{j}+\varepsilon_{j}
$$

The $\varepsilon$ represents the random error and it satisfies the independent and identically distributed condition.

In recent years, several nonparametric regression methods are proposed in order to get a more powerful regression model, such as Nadaraya-Watson method $[15,16]$ and local linear method [17]. In traditional statistical models, the regression function is usually predefined and we just need to find the optimum coefficients of the regression model. However, the nonparametric regression focuses on finding the best model which fits the experimental data the most,. So the nonparametric regression is a sort of data-driven modeling method and it is more potential to model the nonlinear system $[18,19]$.

Even though the nonparametric regression method is potential, its application in the machining field is little. Valentinčič and Junkar [20] applied the nonparametric method to the electrical discharge machining process (EDM) and obtained a valuable result. Munoz-Sánchez et al. [21] studied the inverse identification of material parameters using hybrid FEM/LPR method and FEM/ANN. Local polynomial regression (LPR) is also a kind of nonparametric regression. The result shows that LPR method is better than ANN method, indicating the performance of the nonparametric regression method.

The varying-coefficient model (VCM) [22] is an extension of nonparametric regression. It is based on the linear regression and assumes that the coefficient term is the function of 
another predictor variable $U$ to enhance its flexibility, and the form is

$$
Y=\beta_{1}(U) X_{1}+\beta_{2}(U) X_{2}+\cdots+\beta_{p}(U) X_{p}+\varepsilon .
$$

In the VCM method, the coefficient $\beta_{j}(U)$ is the function of the predictor variable $U$ and is also the coefficient of another predictor variable $X_{j}$. The VCM method concentrates more on $U$ than on $X$. Usually, assume $X_{1} \equiv 1$ to add the intercept term.

The estimation of the VCM method is shown in Fan and Zhang [23]. The main task is to use the sample $\left(Y_{i} ; X_{i 1}, X_{i 2}, \ldots, X_{i p}, U_{i}\right)(i=1,2, \ldots, n)$ to minimize the following function

$$
\begin{aligned}
& \sum_{i=1}^{n}\left\{Y_{i}-\sum_{j=1}^{p}\left(\beta_{j}\left(u_{0}\right)+\beta_{j}^{\prime}\left(u_{0}\right)\left(U_{i}-u_{0}\right)\right) X_{i j}\right\}^{2} \\
& \quad \times K_{h}\left(U_{i}-u_{0}\right)=\mathrm{min},
\end{aligned}
$$

where $K_{h}(t)=K(t / h) / h$ is the kernel function and $h$ is the bandwidth. Gaussian function is a commonly used kernel function, which has the following expression $K(t)=$ $\exp \left(-0.5 t^{2}\right) / \sqrt{2 \pi}$.

Let

$$
\begin{gathered}
X\left(u_{0}\right) \\
=\left[\begin{array}{cccccc}
X_{11} & \cdots & X_{1 p} & X_{11}\left(U_{1}-u_{0}\right) & \cdots & X_{1 p}\left(U_{1}-u_{0}\right) \\
X_{21} & \cdots & X_{2 p} & X_{21}\left(U_{2}-u_{0}\right) & \cdots & X_{2 p}\left(U_{2}-u_{0}\right) \\
\vdots & & \vdots & \vdots & & \vdots \\
X_{n 1} & \cdots & X_{n p} & X_{n 1}\left(U_{n}-u_{0}\right) & \cdots & X_{n p}\left(U_{n}-u_{0}\right)
\end{array}\right], \\
W\left(u_{0}\right) \\
=\operatorname{diag}\left(K_{h}\left(U_{1}-u_{0}\right), K_{h}\left(U_{2}-u_{0}\right), \ldots, K_{h}\left(U_{n}-u_{0}\right)\right), \\
a\left(u_{0}\right)=\left(\beta_{1}\left(u_{0}\right), \beta_{2}\left(u_{0}\right), \ldots, \beta_{p}\left(u_{0}\right), \beta_{1}^{\prime}\left(u_{0}\right),\right. \\
\left.\beta_{2}^{\prime}\left(u_{0}\right), \ldots, \beta_{p}^{\prime}\left(u_{0}\right)\right)^{T} \\
Y=\left(Y_{1}, Y_{2}, \ldots, Y_{n}\right)^{T} .
\end{gathered}
$$

Assuming that $e_{j}$ denotes a $2 p$-dimensional row vector, in which the $j$-th element is one and the other is zero, the estimation result of $\widehat{\beta}_{j}(\cdot)$ is

$$
\begin{aligned}
\widehat{\beta}_{j}\left(u_{0}\right)= & e_{j} a\left(u_{0}\right) \\
= & e_{j}\left(X^{T}\left(u_{0}\right) W\left(u_{0}\right) X\left(u_{0}\right)\right)^{-1} \\
& \times X^{T}\left(u_{0}\right) W\left(u_{0}\right) Y .
\end{aligned}
$$

The bandwidth selection is vital to the nonparametric regression, as shown by Härdle and Mammen [24]. Crossvalidation [25] is a popular method to evaluate the risk of the model, and there are several cross-validation methods,
TABLE 2: The linearity testing result of the transient current amplitude.

\begin{tabular}{cccc}
\hline & Min & Mean & Max \\
\hline$R^{2}$ & 0.8886 & 0.9374 & 0.9793 \\
\hline
\end{tabular}

such as threefold cross-validation, tenfold cross-validation, and leave-one-out cross-validation. However, as shown in [26], the tenfold cross-validation has become the standard method in the practical terms. In this research, the 10 -fold cross-validation is preferred.

From (7), if the predictor variable $X$ is the machining parameters, the predictor variable $U$ is the time $t$, and the response variable is the transient current amplitude, the VCM method would have the same formulation with the transient current predictive model, meaning that the VCM method is suitable to solve this model.

\section{Experiment and Result}

Several experiments are conducted under different spindle speed, and the feed-motor current data has been collected. The details of the experiments are described in Section 2.1. In this section, only the first cycle of the transient current amplitude is analyzed.

4.1. Model Analysis. The transient current predictive model has the same formulation as the VCM method, but the VCM method is based on the linear regression. It is important to test the linearity of predictor variable $\ln I(t)$ and the response variable $\ln S$ in the predictive model.

The samples used in the linearity testing are lain on the same phase in the cycle under different spindle speed level, and the phase $t=0.02 i, i=0,1, \ldots, 50$, as shown in Figure 5. Since the amplitude cycles of all spindle speed level are normalized to 1 , the samples can reflect the law of transient current under different spindle speed, and the linearity testing results of $\ln I(t)$ and $\ln S$ are shown in Figure 6.

Table 2 shows the multiple correlation coefficients $\left(R^{2}\right)$ of the linearity result; it can be clearly seen that the mean of $R^{2}$ is as high as 0.9374 , which indicates that the linearity between $\ln I(t)$ and $\ln S$ is very good. At the same time, the predictive model of the transient current is also validated.

4.2. Results Analysis and Discussion. This section presents the predictive result of VCM method. 600 samples of transient current are randomly selected from the six-level spindle speed. The bandwidth of VCM method is determined using tenfold cross-validation. The results are shown in Figures 7 and 8 .

Figure 7 presents the regression result of the total samples, the regression results match the experiment data well, $R^{2}$ of the total sample is as high as 0.9806 , and the mean relative errors (MRE) are $5.99 \%$, less than 6\%. Figure 8 shows the predicted results under different spindle speed and the results also agree with the experimental result well, which 

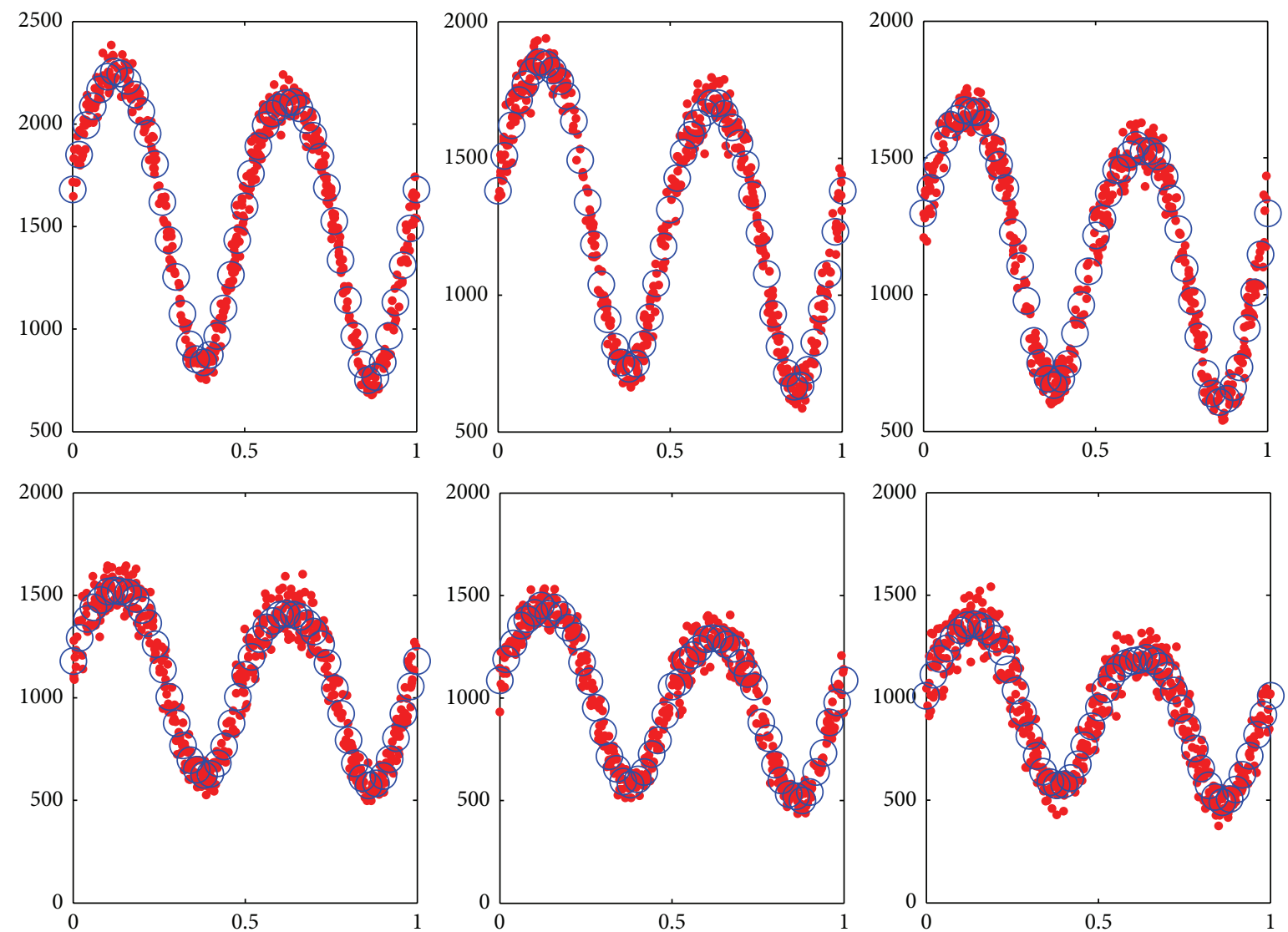

FIgURE 5: The samples used for the linearity testing of the transient current amplitude.

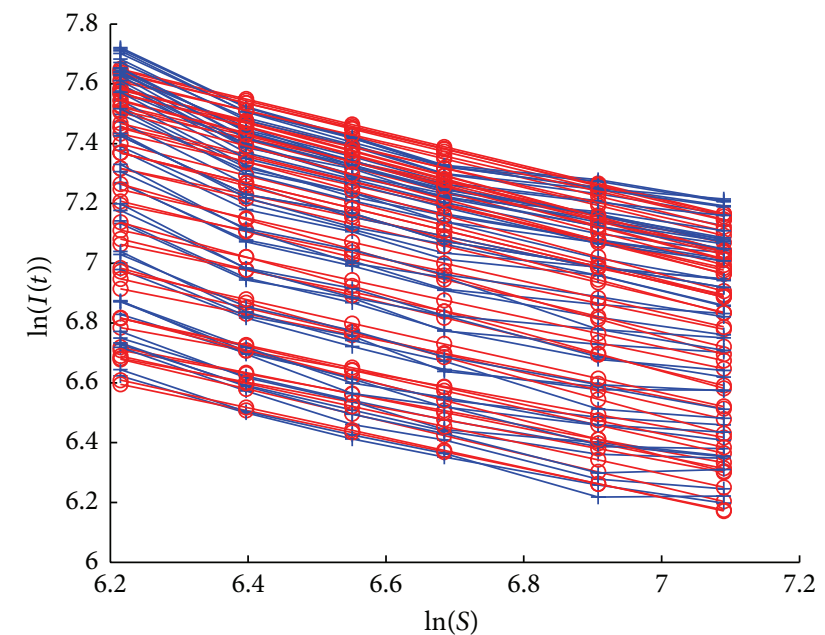

FIGURE 6: The linearity testing results under each sample for the transient current amplitude.

indicates that the VCM method achieves good results at all spindle speed levels.

Table 3 presents the comparison between the VCM method and the Fourier series fitting. The Fourier series fitting is used to fit the transient current amplitude on each

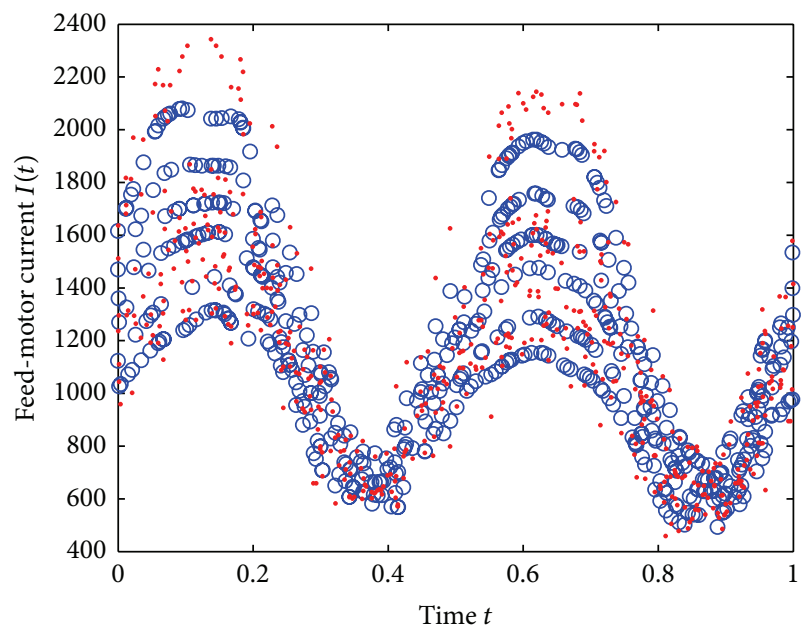

FIGURE 7: The predictive result of VCM method.

level of spindle speed. The testing result shows that all the $R^{2}$ of VCM are much higher than Fourier series fitting, but these MRE are close to each other. The result indicates that VCM has a better performance to fit the transient current amplitude than the Fourier series fitting because the data samples of VCM came from all spindle speed level; the fault tolerance of VCM is better. 

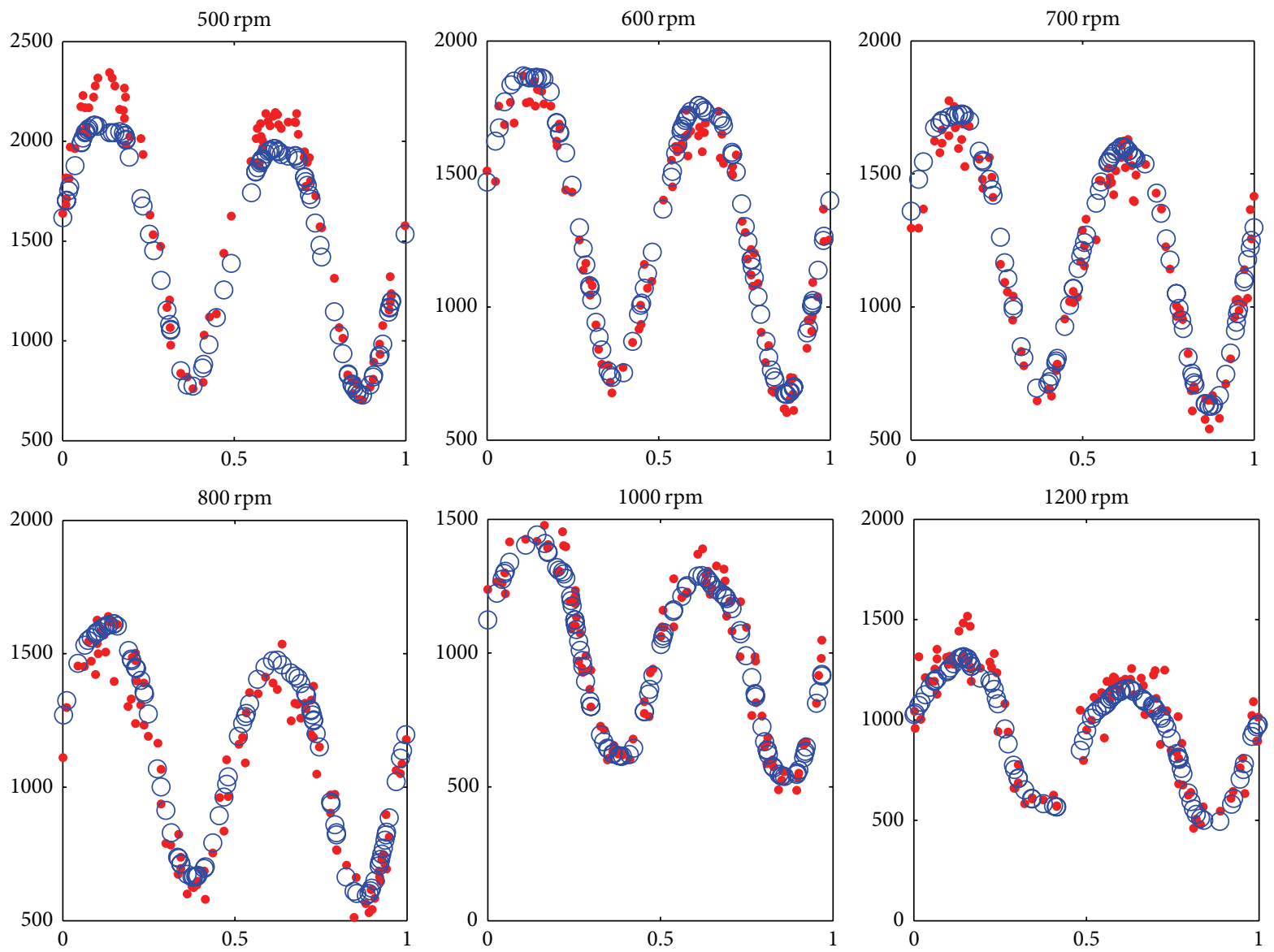

FIGURE 8: The predictive results of the VCM method under each spindle speed.

TABLE 3: The result of VCM method.

\begin{tabular}{lcccccccc}
\hline \multicolumn{2}{c}{ Spindle speed } & & Total & 500 & 600 & 700 & 800 & 1000 \\
\multirow{2}{*}{$\mathrm{VCM}$} & $R^{2}$ & 0.9806 & 0.9910 & 0.9900 & 0.9876 & 0.9763 & 0.9768 & 0.9647 \\
& $\mathrm{MRE}$ & $5.99 \%$ & $6.19 \%$ & $4.64 \%$ & $5.91 \%$ & $6.63 \%$ & $5.41 \%$ & $7.14 \%$ \\
\hline \multirow{2}{*}{ Fourier series } & $R^{2}$ & - & 0.9690 & 0.9641 & 0.9548 & 0.9348 & 0.9378 & 0.8924 \\
& $\mathrm{MRE}$ & - & $5.36 \%$ & $5.21 \%$ & $5.80 \%$ & $6.76 \%$ & $6.71 \%$ & $8.56 \%$ \\
\hline
\end{tabular}

Figure 9 presents the result of the coefficient parameters $\beta_{0}(t), \beta_{1}(t)$ of VCM method. $\beta_{0}(t), \beta_{1}(t)$ are also the coefficient parameters $\ln K_{0}(t), b_{1}(t)$ of the transient current predictive model. The curves of both coefficients have a big change at the point of 0.4 and 0.9 , meaning that the transient current amplitudes are changing over time.

From the predictive results and linearity testing, the correctness of the transient current predictive model is validated and the performance of the VCM methods is demonstrated. The predicted results of the transient current amplitude are good and fit the experiment data very well.

\section{Conclusion and Future Work}

The feed-motor current has a wide influence on other cutting variables, such as the cutting force and the tool wear, but there are few studies on the feed-motor current. This paper focuses on the transient current of the stable milling process and established a predictive model for the transient current amplitude. In this model, the change regulation of the transient current amplitude and the effect of the spindle speed to the transient current amplitude are considered. The VCM method is applied to solve this model, because the predictive model is a typical panel data type. Several experiments are conducted to verify the correctness of the predictive model; the results show that VCM method has achieved a good result and the predictive values match the experimental value well.

The main contribution of this paper is the model on the transient current amplitude, but we only focus on the stable milling process. Future works would be done to investigate the relationship between machining parameters and other physical variables in the milling process. As the transient current amplitude is easily interfered by environment factors, 


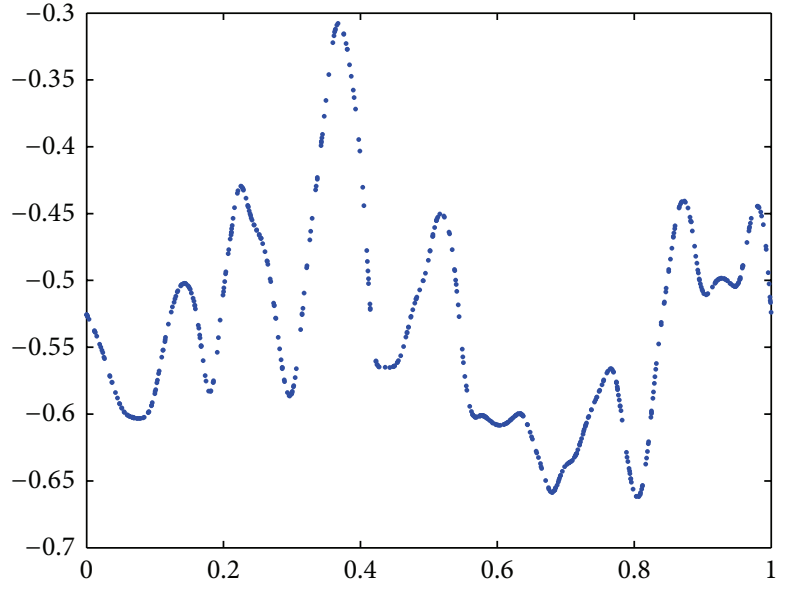

(a) The curve of $\beta_{0}(t)$

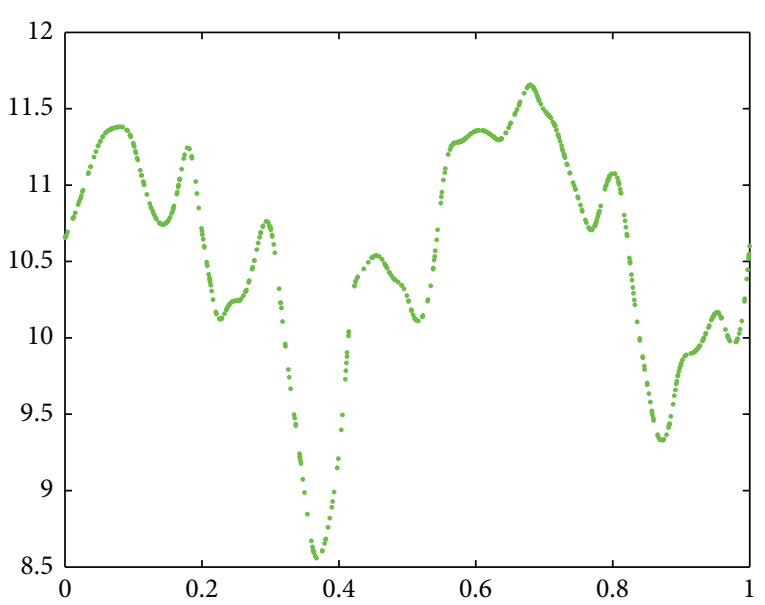

(b) The curve of $\beta_{1}(t)$

FIGURE 9: The result of the coefficient parameter of VCM.

the analysis of this disturbance based on VCM method is also important to find more properties of the transient current.

\section{Conflict of Interests}

The authors declare that there is no conflict of interests regarding the publication of this paper.

\section{Acknowledgments}

This research work is supported by the National Basic Research Program of China (973 Program) under Grant no. 2011CB706804 and the Natural Science Foundation of China (NSFC) under Grants nos. 51435009 and 51305147.

\section{References}

[1] D. Cica, B. Sredanovic, G. Lakic-Globocki, and D. Kramar, "Modeling of the cutting forces in turning process using various methods of cooling and lubricating: an artificial intelligence approach," Advances in Mechanical Engineering, vol. 2013, Article ID 798597, 8 pages, 2013.

[2] G. Quintana and J. Ciurana, "Chatter in machining processes: a review," International Journal of Machine Tools and Manufacture, vol. 51, no. 5, pp. 363-376, 2011.

[3] M. Rizal, J. A. Ghani, M. Z. Nuawi, and C. H. C. Haron, "Online tool wear prediction system in the turning process using an adaptive neuro-fuzzy inference system," Applied Soft Computing Journal, vol. 13, no. 4, pp. 1960-1968, 2013.

[4] M. Chandrasekaran, M. Muralidhar, C. M. Krishna, and U. S. Dixit, "Application of soft computing techniques in machining performance prediction and optimization: a literature review," International Journal of Advanced Manufacturing Technology, vol. 46, no. 5-8, pp. 445-464, 2010.

[5] M. Moghri, M. Madic, M. Omidi, and M. Farahnakian, "Surface roughness optimization of polyamide-6/nanoclay nanocomposites using artificial neural network: genetic algorithm approach," The Scientific World Journal, vol. 2014, Article ID 485205, 7 pages, 2014.
[6] Q. Ren, M. Balazinski, K. Jemielniak, L. Baron, and S. Achiche, "Experimental and fuzzy modelling analysis on dynamic cutting force in micro milling," Soft Computing, vol. 17, no. 9, pp. 1687-1697, 2013.

[7] J. Gokulachandran and K. Mohandas, "Comparative study of two soft computing techniques for the prediction of remaining useful life of cutting tools," Journal of Intelligent Manufacturing, 2013.

[8] M. V. Cakir, O. Eyercioglu, K. Gov, M. Sahin, and S. H. Cakir, "Comparison of soft computing techniques for modelling of the EDM performance parameters," Advances in Mechanical Engineering, vol. 2013, Article ID 392531, 15 pages, 2013.

[9] I. Zohourkari, M. Zohoor, and M. Annoni, "Investigation of the effects of machining parameters on material removal rate in abrasive waterjet turning," Advances in Mechanical Engineering, vol. 2014, Article ID 624203, 11 pages, 2014.

[10] A. B. Sharkawy, "Prediction of surface roughness in end milling process using intelligent systems: a comparative study," Applied Computational Intelligence and Soft Computing, vol. 2011, Article ID 183764, 18 pages, 2011.

[11] S. Aggarwal, N. Nešić, and P. Xirouchakis, "Cutting torque and tangential cutting force coefficient identification from spindle motor current," International Journal of Advanced Manufacturing Technology, vol. 65, no. 1-4, pp. 81-95, 2013.

[12] Y. H. Jeong and D. W. Cho, "Estimating cutting force from rotating and stationary feed motor currents on a milling machine," International Journal of Machine Tools and Manufacture, vol. 42, no. 14, pp. 1559-1566, 2002.

[13] D. Kim and D. Jeon, "Fuzzy-logic control of cutting forces in CNC milling processes using motor currents as indirect force sensors," Precision Engineering, vol. 35, no. 1, pp. 143-152, 2011.

[14] M. Xu and C. Jie, "Research on the relationship between CNC machine tool spindle current and cutting parameters," Machinery, vol. 12, pp. 28-30, 2010.

[15] G. Dudek, "Tournament searching method for optimization of the forecasting model based on the Nadaraya-Watson estimator," Artificial Intelligence and Soft Computing, vol. 8468, pp. 339-348, 2014. 
[16] H. Long and L. Qian, "Nadaraya-Watson estimator for stochastic processes driven by stable Lévy motions," Electronic Journal of Statistics, vol. 7, pp. 1387-1418, 2013.

[17] M. Avery, Literature Review for Local Polynomial Regression, 2013, http://www4.ncsu.edu/ mravery/AveryReview2.pdf.

[18] A. Donnelly, B. Misstear, and B. Broderick, "Application of nonparametric regression methods to study the relationship between $\mathrm{NO}_{2}$ concentrations and local wind direction and speed at background sites," Science of the Total Environment, vol. 409, no. 6, pp. 1134-1144, 2011.

[19] F. Li, T. Yan, and L. Su, "Solution of an integral-differential equation arising in oscillating magnetic fields using local polynomial regression," Advances in Mechanical Engineering, vol. 2014, Article ID 101230, 9 pages, 2014.

[20] J. Valentinčič and M. Junkar, "Detection of the eroding surface in the EDM process based on the current signal in the gap," The International Journal of Advanced Manufacturing Technology, vol. 28, no. 3-4, pp. 294-301, 2006.

[21] A. Munoz-Sánchez, I. M. González-Farias, X. Soldani, and M. H. Miguélez, "Hybrid FE/ANN and LPR approach for the inverse identification of material parameters from cutting tests," International Journal of Advanced Manufacturing Technology, vol. 54, no. 1-4, pp. 21-33, 2011.

[22] B. U. Park, E. Mammen, Y. K. Lee, and E. R. Lee, "Varying coefficient regression models: a review and new developments," International Statistical Review, 2013.

[23] J. Fan and W. Zhang, "Statistical methods with varying coefficient models," Statistics and Its Interface, vol. 1, no. 1, pp. 179-195, 2008.

[24] W. Härdle and E. Mammen, "Comparing nonparametric versus parametric regression fits," The Annals of Statistics, vol. 21, no. 4, pp. 1926-1947, 1993.

[25] S. Arlot and A. Celisse, "A survey of cross-validation procedures for model selection," Statistics Surveys, vol. 4, pp. 40-79, 2010.

[26] I. H. Witten, E. Frank, and M. A. Hall, Data Mining: Practical Machine Learning Tools and Techniques, China Machine Press, Beijing, China, 3rd edition, 2012. 


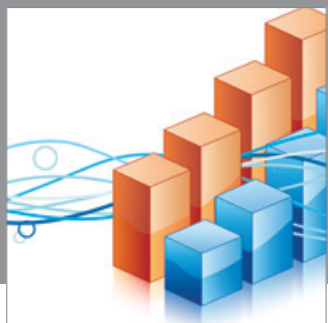

Advances in

Operations Research

mansans

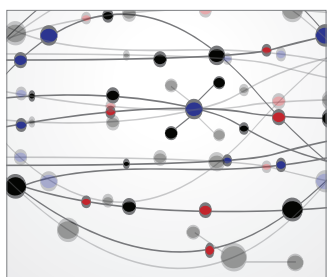

The Scientific World Journal
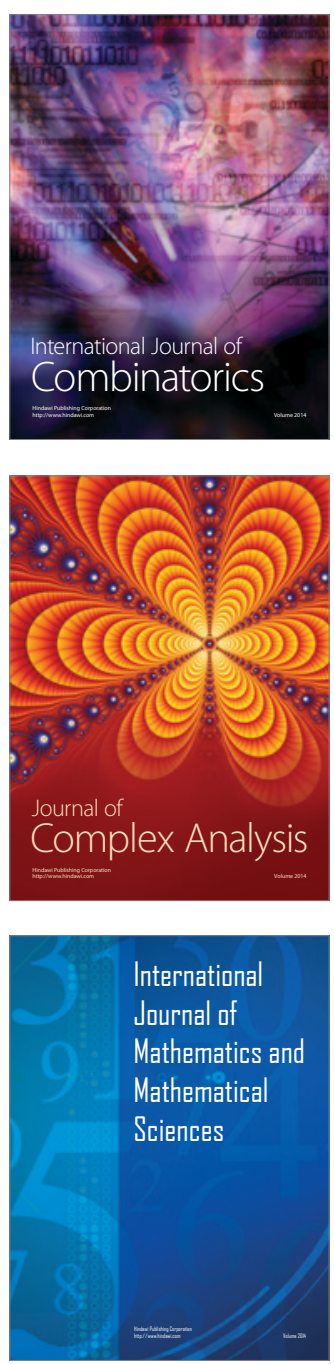
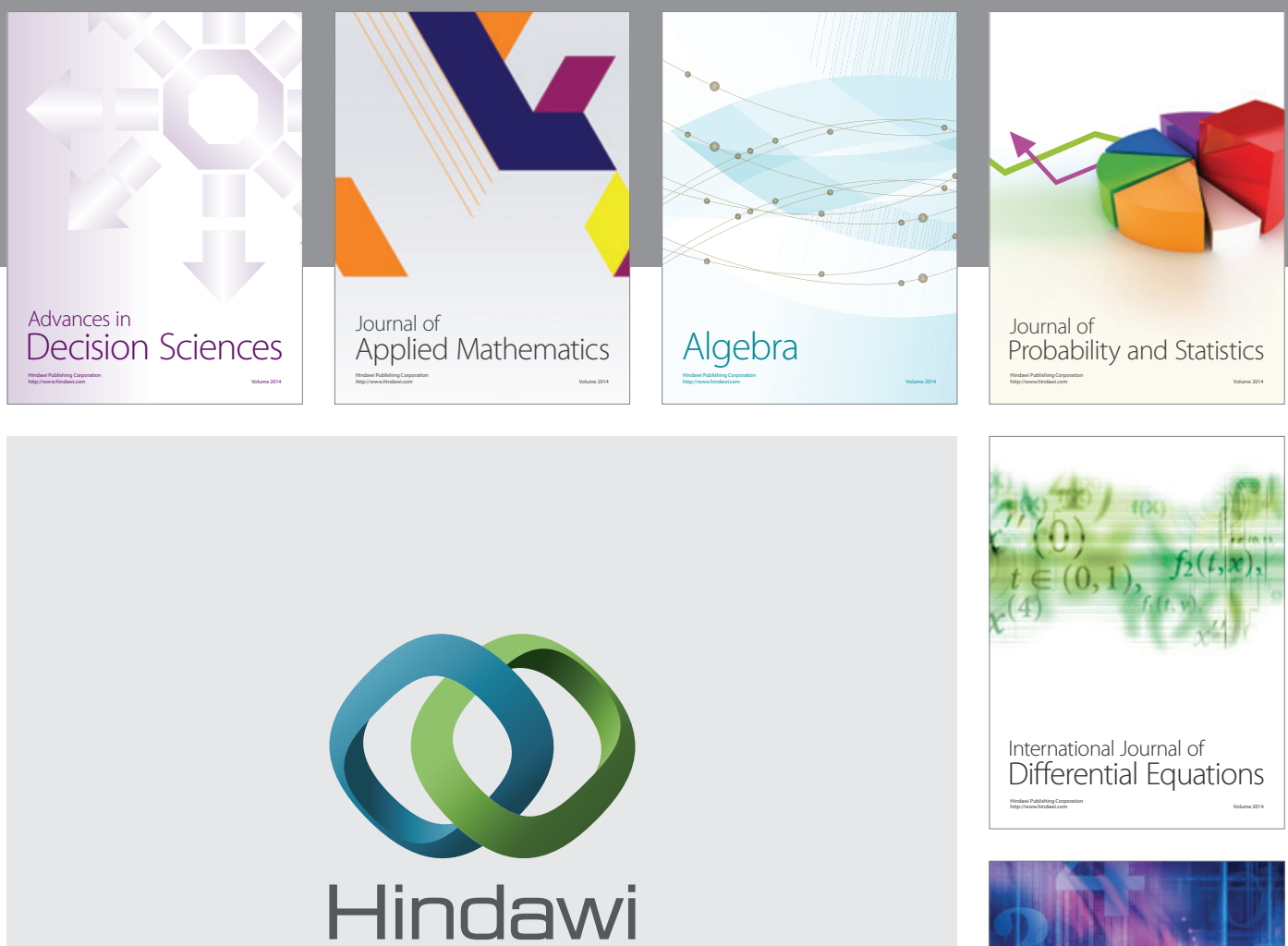

Submit your manuscripts at http://www.hindawi.com
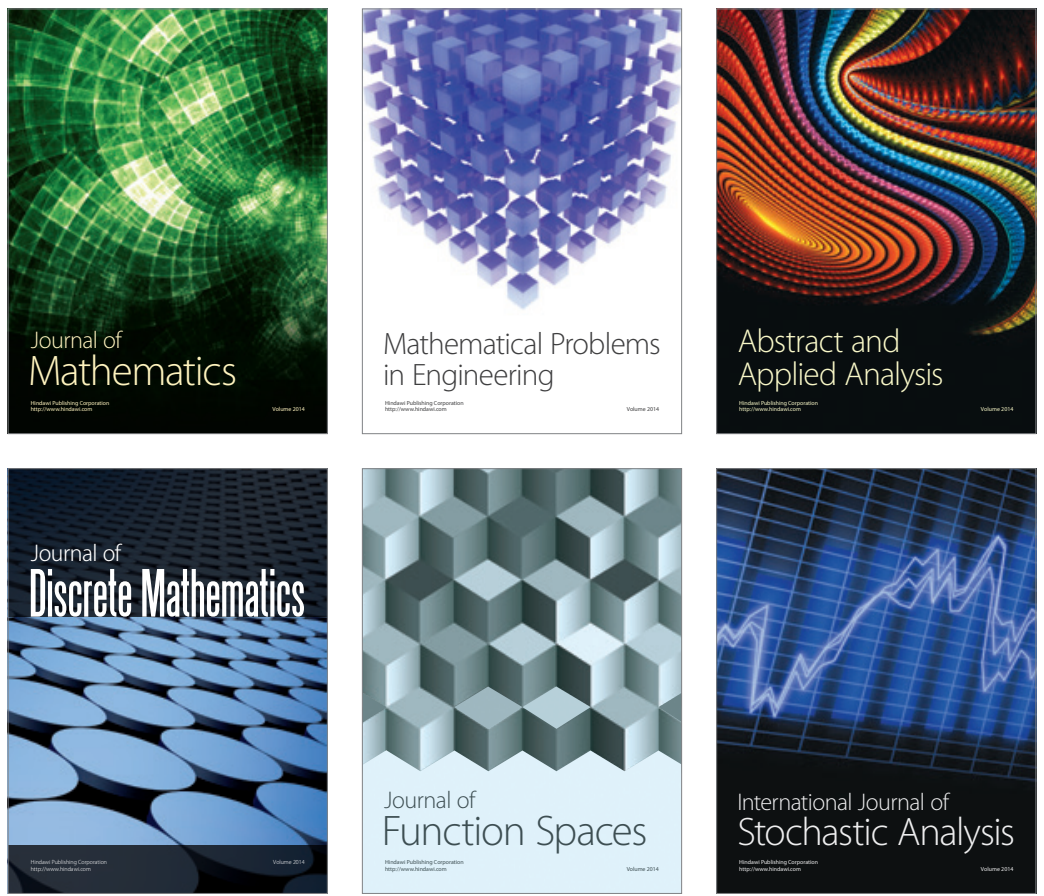

Journal of

Function Spaces

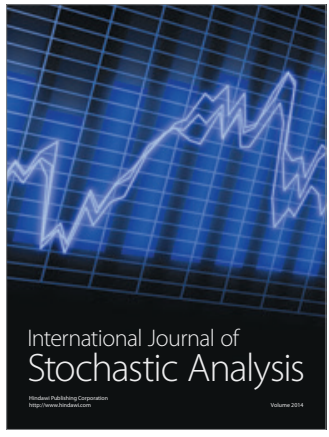

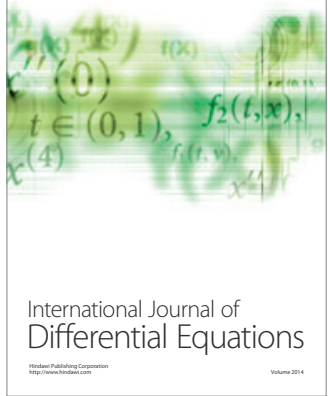
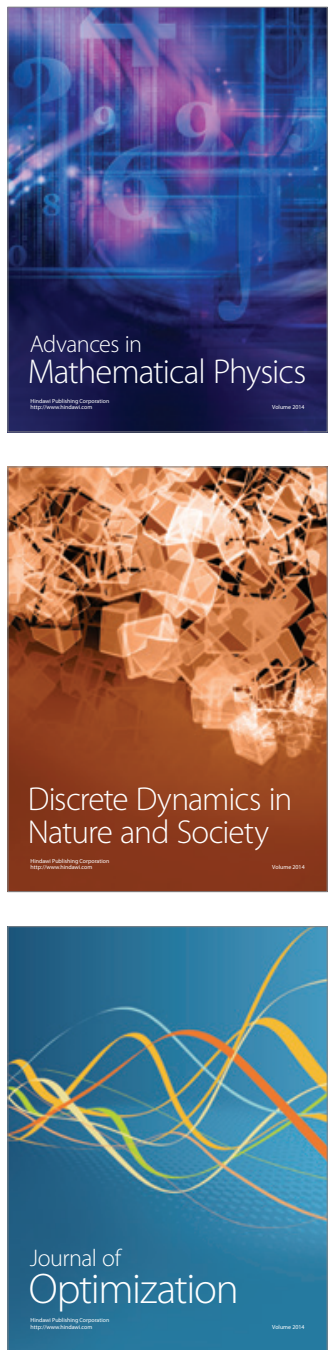